\title{
DIFFUSION, MERIDIONAL CIRCULATION AND MASS LOSS IN MAIN SEQUENCE AND HORIZONTAL BRANCH STARS
}

\author{
G. MICHAUD \\ Département de Physique \\ Université de Montréal \\ C.P. 6128, succursale $A$ \\ Montréal, Qué., H3C 3J7 \\ Canada
}

\begin{abstract}
Photospheric abundances are used to determine the importance of atomic diffusion, meridional circulation, mass loss and turbulence in main sequence and horizontal branch stars. Atomic diffusion leads to the appearance of the Li gap and the AmFm and $\mathrm{HgMn}$ phenomena at approximately the $\mathrm{T}_{\text {eff }}$ at which they are observed. It leads to a 12 to $25 \%$ reduction in the age of halo stars. The $\mathrm{Li}$ abundance in Halo stars is probably $50 \%$ of the original abundance. Atomic diffusion competes with meridional circulation. The $V \sin i$ at which the HgMn and AmFm phenomena disappear give an argument in favour of the meridional circulation model of Tassoul and Tassoul. Mass loss is probably present in AmFm stars and cooler $\mathrm{F}$ stars but only at the rate of $10^{-15} \mathrm{M}_{\odot} \mathrm{yr}^{-1}$. In many objects, the turbulent particle diffusion coefficient is at most 10 times larger than the atomic diffusion coefficient.
\end{abstract}

\section{Context}

Over the last few years the quality of observational material has improved to the point that much finer tests of stellar evolution models have become possible. One example is the dip in $\mathrm{Li}$ abundance at $\mathrm{T}_{\text {eff }}=6700 \mathrm{~K}$ reviewed by Rebolo elsewhere in this volume. Demonstrating the presence of the Li gap has been made possible by the high signal to noise data available on relatively faint objects.

We develop tests of the physical processes using the observed abundance anomalies. In addition to "standard" stellar physics, we shall consider particle transport by atomic diffusion, meridional circulation, mass loss and turbulence. Atomic diffusion is a basic physical process that must be included unless there are more efficient transport processes that compete with it and wipe out its effects. Meridional circulation is calculated from first principles without arbitrary parameters (Tassoul and Tassoul 1982); it defines a model for particle transport in rotating stars whose effects can be tested. Mass loss is known to be present in the Sun at a rate of $10^{-14} \mathrm{M}_{\odot} \mathrm{yr}^{-1}$ and it is appropriate to determine what would be its effect in other stars at that level. While mass loss is a natural phenomenon that may well play a role, its introduction currently requires at least one arbitrary parameter. As to turbulence, it is discussed elsewhere in this volume and we shall mention it only in so far as it is possible to put interesting upper limits on it. 


\section{Physical Processes}

Tassoul and Tassoul (1982) obtained the meridional circulation velocity patterns in the radiative zones of Cowling models with Kramer or electron scattering opacities. Charbonneau, Michaud and Proffitt (1989), using the method developed by Tassoul and Tassoul (1982), determined meridional circulation patterns in realistic evolutionary stellar models (see their Fig. 3) by assuming that the convection zone acted as a boundary. In their solution, there is no circulation in the convection zone itself and below a relatively thin boundary layer, the circulation velocities are close to those obtained in the absence of a convection zone. If this layer is sufficiently turbulent, it links the abundances in the convection zones to those in the circulation patterns below. If it is not turbulent, the convection zone could be partly decoupled from the abundances in circulation patterns and the settling from the convection zone might not be so strongly affected by meridional circulation. The extent to which meridional circulation penetrates the convection zone and the turbulence of the boundary layer below the convection zone are uncertain. The results presented below were obtained assuming that meridional circulation penetrates the convection zone and so maximize its effect.

The mass loss rate, $\mathrm{dM} / \mathrm{dt}$, is assumed small enough to have negligible effect on stellar structure. It merely introduces, throughout the static stellar envelope, a global outward velocity of matter, $\mathbf{v}_{\mathbf{w}}$ :

$$
\frac{d M}{d t}=-4 \pi R^{2} \rho v_{w}
$$

This equation expresses flux conservation for the main constituent.

A trace element diffusing in the presence of mass loss must satisfy the conservation equation:

$$
-\mathrm{N}_{\mathrm{H}} \frac{\delta \mathrm{C}}{\delta \mathrm{t}}=\frac{1}{\mathrm{r}^{2}} \frac{\delta}{\delta \mathrm{r}}\left(\mathrm{r}^{2} \mathrm{CN}_{\mathrm{H}}\left(\mathrm{v}_{\mathrm{W}}+\mathrm{v}_{\mathrm{D}}\right)\right)
$$

where $c$ is the concentration $\left(c=n(A) / N_{H}\right)$ and $v_{D}$ is the diffusion velocity of a trace element of atomic mass $A$ and charge $Z$ in ionized hydrogen:

$$
\mathrm{v}_{\mathrm{D}}=-\mathrm{D}_{12}\left\{\frac{\delta \ln \mathrm{c}}{\delta \mathrm{r}}+\left[\left(\mathrm{A}-\frac{\mathrm{Z}}{2}-\frac{1}{2}\right) \mathrm{g}-\mathrm{Ag}_{\mathrm{R}}\right] \frac{\mathrm{m}_{\mathrm{p}}}{\mathrm{kT}}-\mathrm{k}_{\mathrm{T}} \frac{\delta \ln \mathrm{T}_{\mathrm{T}} \mathrm{r}}{\delta}\right\}
$$

The first term on the right hand side is the "classical" diffusion term. It is 0.0 at the beginning of stellar evolution and seldom becomes important in stars since the equilibrium abundance gradients are rarely approached. The second term is the gravitational settling term modified for the presence of the electric field. It is followed by radiative acceleration and thermal diffusion. An equation valid for any binary mixture and in presence of degeneracy is given in Pelletier et al. (1986). A form for a ternary mixture, useful when two species have large abundances, is given by Proffitt and Michaud (1991; see also Loeb, Bahcall and Milgrom 1989). Effects of partial ionization are discussed in Michaud and Babel (1991) and Babel and Michaud (in preparation).

The diffusion coefficients can be found in Paquette et al. (1986). Contrary to those of Chapman and Cowling (1970), they take into account that stellar plasmas are not very dilute plasmas. The corrections are important in the interior of main sequence stars. While these are 
to be preferred to those of Chapman and Cowling, there remain uncertainties specially for the thermal diffusion coefficient (see Michaud 1991). For Li in F stars, the thermal diffusion term is at most $20 \%$ of the gravitational settling term.

The competition between radiative acceleration $\left(\mathrm{g}_{\mathrm{R}}\right)$ and gravity determines whether diffusion leads to over- or underabundances. Accurate radiative accelerations have only been calculated for few elements. General formulae are available (Michaud et al. 1976; Alecian and Artru 1990) but are accurate to at most a factor of 3 (see Michaud 1987 for a discussion). Alecian and Artru (1990) have redetermined radiative accelerations for stellar interiors treating atomic configurations in more detail than Michaud et al. (1976); they obtain larger $\mathrm{g}_{\mathrm{R}}$ when the element mainly has unsaturated lines but smaller $g_{R}$ when the element is abundant enough for its strongest lines to be heavily saturated. The radiative acceleration is mainly determined by the state of ionization of the specie. Radiative accelerations for $\mathrm{Li}$ and $\mathrm{Be}$ are shown on Fig. 4.1 of Michaud and Charbonneau (1990) in the interior of a $T_{\text {eff }}=6700 \mathrm{~K}$ main sequence star. Deep in the interior, $\mathrm{Li}$ is completely ionized. It then has no lines and its radiative acceleration is negligible. Around $200000 \mathrm{~K}, \mathrm{Li}$ is more often in the hydrogenoid state, $\mathrm{Li}$ III. Its radiative acceleration is then about equal to gravity. At cooler $\mathrm{T}$, a larger fraction of $\mathrm{Li}$ is $\mathrm{Li}$ III and $\mathrm{g}_{R}$ increases until $\mathrm{Li}$ gets into the He-like state (Li II) where $\mathrm{g}_{R}$ starts decreasing again. It has been noted by Vauclair (1987) that the presence of a strong line of an abundant element at the frequency of one of the two or three most important lines of $\mathrm{Li}$ could reduce the radiative acceleration on $\mathrm{Li}$. This could widen the gap slightly and make it deeper. No good candidate line is known.

In the absence of calculations from first principles, various phenomenological parametrisations of turbulent diffusion coefficients, $D_{T}$, have been proposed to evaluate the potential effects of turbulence (Schatzman 1969). One calculates the time evolution of the abundance of a given chemical specie, by solving the transport equation including both atomic and turbulent diffusion:

$$
\begin{aligned}
& \rho r^{2} \frac{\delta c}{\delta t}=\frac{\delta}{\delta r}\left\{\rho r^{2}\left\{\left(D_{12}+D_{T}\right) \frac{\delta C}{\delta r}+C D_{12}\left[\left[\left(A-\frac{Z}{2}-\frac{1}{2}\right) g-A g_{R}\right] \frac{m_{p}}{k T}-k_{T} \frac{\delta l n T}{\delta r}\right]\right\}\right\} \\
& -\operatorname{cor} r^{2} s(\rho, T) \text {, }
\end{aligned}
$$

where $c$ is the concentration of the element under study, and $S(\rho, T)$ is a source/sink term usually due to nuclear burning. The other terms are the same as in eq. (3); the latitudinal variation of $D_{T}$ has been neglected (see Charbonneau and Michaud 1991). It is often arbitrarily assumed that turbulence wipes out the effects of atomic diffusion and that $D_{12}$ can be neglected.

\section{Evolution with diffusion}

The parameter free evolutionary models for non-rotating stars assumes that, as the star forms, the abundances are homogeneous and equal to those of the interstellar cloud from which it formed. There are superficial hydrogen and helium convection zones. As evolution proceeds, helium settles below the He II convection zone, which disappears after some $10^{6} \mathrm{yr}$ if $\mathrm{T}_{\text {eff }}>$ $8000 \mathrm{~K}$ (Vauclair Vauclair and Pamjatnikh 1974; Michaud et al. 1976). The chemical separation then goes on below the $\mathbf{H}$ convection zone. Most heavy elements are pushed by radiative acceleration from the radiative envelope into the convection zone and can become 
overabundant. For $\mathrm{T}_{\text {eff }}<8000 \mathrm{~K}$, special care must be taken to properly treat the effect of $\mathrm{He}$ settling on stellar structure. For predicting abundance anomalies, the exact position of the bottom of the convection zone is critical when radiative accelerations vary with temperature as rapidly as they do for $\mathrm{Li}$. One must take into account the effect of He settling and evolution on $T_{\text {eff }}$ (Richer, Michaud, Proffitt 1991). Helium settling reduces the $T_{\text {eff }}$ by about $100 \mathrm{~K}$ at $T_{\text {eff }}$ $=7000 \mathrm{~K}$ and below; as the $T_{\text {eff }}$ goes from 6900 to $6800 \mathrm{~K}$ the mass in the convection zone increases by a factor of 30 . At still cooler $T_{\text {eff }}(<6400 \mathrm{~K})$, the effect of settling on both $T_{\text {eff }}$ and the central He content must be followed to obtain accurate evolutionary tracks (Proffitt and Michaud 1991).

Detailed evolutionary models for AmFm stars must take this variation into account. It is most important below $8000 \mathrm{~K}$. Above that $\mathrm{T}_{\text {eff }}$, the $\mathrm{T}$ at the bottom of the $\mathrm{H}$ convection zone varies less rapidly as a function of $\mathrm{T}_{\text {eff }}$ (Richer, Michaud and Proffitt 1991). However, a star with $\mathrm{T}_{\text {eff }}=9000 \mathrm{~K}$ on the zero age main sequence spends about $30 \%$ of its main sequence life with $\mathrm{T}_{\text {eff }}<8000 \mathrm{~K}$.

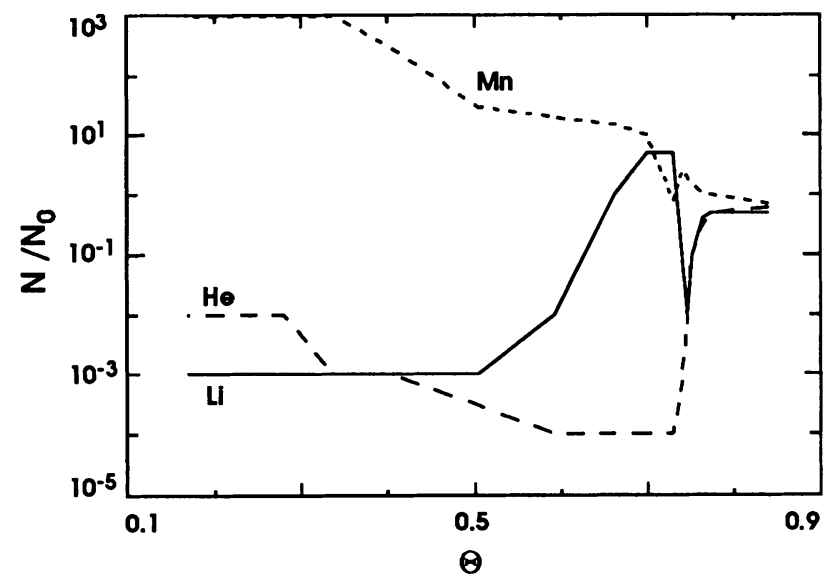

Figure 1. Expected abundances of $\mathrm{He}, \mathrm{Li}$ and $\mathrm{Mn}$ as a function of $\theta\left(=5040 / \mathrm{T}_{\text {eff }}\right)$ in main sequence evolutionary models, at half the main sequence life time. The expected abundance is divided by the original abundance, $\mathrm{N}_{\mathrm{o}}$. Atomic diffusion is included. Around $6000 \mathrm{~K}$, there is settling by a factor of order two for $\mathrm{Li}$ and $\mathrm{He}$ and 1.5 for $\mathrm{Mn}$. As the $\mathrm{T}_{\text {eff }}$ increases, the settling increases for both $\mathrm{Li}$ and $\mathrm{He}$, the $\mathrm{Li}$ gap appearing within about $150 \mathrm{~K}$ of the $\mathrm{T}_{\text {eff }}$ at which it is observed. As the $\mathrm{T}_{\text {eff }}$ further increases, the $\mathrm{Li}$ abundance is largest around $\theta=$ 0.75. It decreases again at higher $T_{\text {eff }}$. The radiative acceleration on $\mathrm{Mn}$ increases, leading to a small overabundance (a factor of 2) around $6800 \mathrm{~K}$. The $\mathrm{Mn}$ abundance then decreases due to the $g_{R}$ on $\mathrm{Mn}$ decreasing as it gets in the rare gas configuration below the convection zone, but the abundance increases considerably for $\theta<0.75$, the Am star domain. Large overabundances are expected in the Am star domain $(0.5<\theta<0.75)$. They are even larger for $\mathrm{T}_{\text {eff }}>10000 \mathrm{~K}$, where HgMn stars are observed (Alecian and Michaud 1981). These calculations involve no arbitrary parameter, once the mixing length is fixed by the solar model. Calculating precisely the $\mathrm{T}_{\text {eff }}$ at which the AmFm and Li gap phenomena are expected in evolutionary models and determining precisely the $T_{\text {eff }}$ at which these phenomena are observed would then be a critical test for stellar evolution. 
Surface abundances predicted from parameter free evolutionary models of non-rotating stars are shown on Fig. 1 for $\mathrm{He}, \mathrm{Li}$ and $\mathrm{Mn}$. Some of the points appearing on the figure come from detailed calculations while others are estimates. Given the uncertainties of the observational and theoretical $T_{\text {eff }}$ scales, theory explains simultaneously the $T_{\text {eff }}$ of the disappearance of the $\mathrm{AmFm}$ phenomenon and the $\mathrm{T}_{\text {eff }}$ of the $\mathrm{Li}$ gap. It should be possible to reduce the uncertainties of both and obtain a very critical test of main sequence models.

\subsection{UPPER MAIN SEQUENCE AND AM STARS}

Evolutionary calculations taking diffusion into account predict, over the interval of the Am phenomenom, $10000>\mathrm{T}_{\text {eff }}>8000 \mathrm{~K}$, large overabundances, at least by two orders of magnitude for most heavy elements such as rare earth and iron peak elements. Calcium and scandium become underabundant only after the disappearance of the He II convection zone, since they are supported by radiative acceleration below that convection zone but not below the $\mathrm{H}$ convection zone. Since underabundances of $\mathrm{Ca}$ and $\mathrm{Sc}$ are essential observational characteristics of most Am stars, the disappearance of the He II convection zone is essential if Am stars are to be explained by this diffusion model. As the results of Michaud et al. (1976) show, most of the elements for which $g_{R}>g$ below the $H$ convection zone are observed to be overabundant and those for which $g_{R}<g$ are underabundant.

For some elements, the conclusions are uncertain because $g_{R}$ is too close to $g$ and it is uncertain which way diffusion should go below the $\mathrm{H}$ convection zone. This is in particular the case for $\mathrm{Fe}$ which has a very large number of lines, but also a large abundance, and for which high accuracy $g_{R}$ calculations are needed.

The anomalies are predicted to be much larger than observed for most elements. Furthermore, while the observed anomalies vary considerably at a given $T_{\text {eff }}$, they are all predicted to be very large in the parameter free diffusion model, specially at $T_{\text {eff }}>8500 \mathrm{~K}$.

\subsection{THE F STARS}

The disappearance of the AmFm phenomenon for $T_{\text {eff }}<7000 \mathrm{~K}$ is caused by the increasing mass in the convection zone (Fig. 2a of Michaud 1986) coupled to the increasing $T$ at the bottom of the convection zone. As $\mathrm{T}_{\text {eff }}$ decreases, diffusion processes slow down since the helium settling time scale, $\theta$, varies approximately as (Michaud 1977):

$\theta=2.310^{11} \Delta \mathrm{M}^{0.545}(\mathrm{yr})$

where $\Delta \mathrm{M}$ is the mass in the convection zone in solar mass units. The settling time scale of helium is given in Fig. 5 of Michaud et al. (1983). The diffusion time scale becomes longer than the evolutionary time scale for some $T_{\text {eff }}$ around $6300 \mathrm{~K}$, the exact value depending on the mixing length, $\alpha$. The evolutionary time scale is then too short to allow large overabundances or underabundances to materialize. Since, at $\mathrm{T}_{\text {eff }}=6500 \mathrm{~K}$, the radiative accelerations become about equal to gravity below the convection zone for a number of elements (Michaud et al. 1976), the upwards diffusion is further reduced. The temperature at the bottom of the $\mathrm{H}$ convection zone becomes large enough that $\mathrm{Ca}$ and $\mathrm{Sc}$ lose one more electron, leave the noble gas configuration and are supported by radiative acceleration, so that they do not settle gravitationnally. All these factors conspire to cause the disappearance of the AmFm phenomenon. 


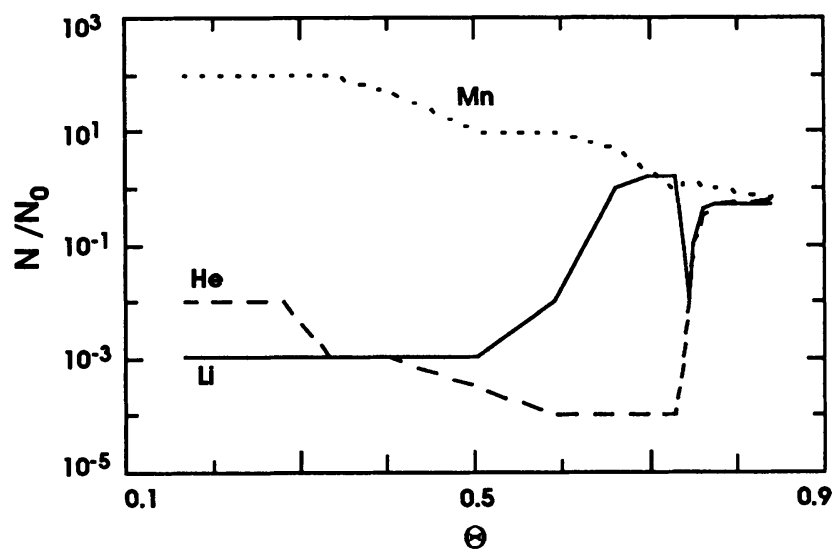

Figure 2. Same as Fig. 1 but in the presence of a mass loss rate of $10^{-15} \mathrm{M}_{\odot} \mathrm{yr}^{-1}$, ten times smaller than the solar mass loss rate. Such a mass loss rate does not modify the Li gap. It decreases the $\mathrm{Mn}$ overabundance expected at the $\mathrm{T}_{\text {eff }}$ of the gap. It reduces the overabundances expected for Mn to approximately the observed values in AmFm stars. It is too small to have an effect on the $\mathrm{He}$ abundance of He stars. A larger mass loss rate is needed to cause $\mathrm{He}$ overabundances.

The surprise in those stars came with the observation of the Li abundance gap (see Rebolo, this volume; also Michaud and Charbonneau 1990 for a review). In the Hyades, at $T_{\text {eff }}$ $=6700 \mathrm{~K}$, there is a dip of nearly two orders of magnitude in the $\mathrm{Li}$ abundance. The gap is only $300 \mathrm{~K}$ wide and is well defined. In other clusters it is not so well defined but this is partly caused by larger observational errors (see Michaud and Charbonneau 1990). The Li gap could be the cool $\mathrm{T}_{\text {eff }}$ continuation of the AmFm phenomenon (Michaud 1986).

At $\mathrm{T}_{\text {eff }}>6900 \mathrm{~K}, \mathrm{Li}$ is supported by its radiative acceleration below the convection zone. As the $T_{\text {eff }}$ decreases, the depth of the convection zone increases, so does the $T$ at its bottom and $\mathrm{Li}$ becomes completely ionized; with no electron left, its radiative acceleration becomes negligible. Lithium then settles gravitationally for $\mathrm{T}_{\text {eff }}<6900 \mathrm{~K}$ (see Fig 4.2 of Michaud and Charbonneau 1990). As the $T_{\text {eff }}$ further decreases and the convection zone becomes more massive, the diffusion time scale increases. At the age of the Hyades, the shape of the $\mathrm{Li}$ gap is reasonably well reproduced, except that the $\mathrm{Li}$ abundance is predicted to be larger than observed for $T_{\text {eff }}>7000 \mathrm{~K}$.

\subsection{HALO STARS}

Atomic diffusion has also been shown to play a major role in globular cluster and field halo stars. Since turbulence transports the photospheric $\mathrm{Li}$ from the surface to a temperature where it burns, the observed $\mathrm{Li}$ abundance limits the maximum value of turbulence (Proffitt and Michaud 1991). Even with the maximum allowed turbulence, evolutionary calculations give a reduction of the age of globular clusters by some $15 \%$ due to the gravitational settling of $\mathrm{He}$ (see their Fig. 4). The abundance of $\mathrm{Li}$ is also reduced by a factor of nearly 2 . Turbulence is not needed to reproduce the observations but the observations can be reproduced only if 
turbulence is very small. These results differ from those of Delyannis et al. (1990) mainly in that these authors had neglected both the contribution of thermal diffusion and the effect of $\mathrm{He}$ settling on structure.

Introducing enough turbulence in the center to stop gravitational settling, modifies the abundance gradients that nuclear burning leads to and so the evolution; the effect of diffusion on evolution seems unavoidable.

These calculations used the best diffusion coefficients available (Paquette et al. 1986). The main uncertainty in the preceding conclusion comes from uncertainties in the value of these coefficients (see Michaud 1991 for a discussion).

\section{Mass loss}

On Fig. 2 is shown the effect of a mass loss of $10^{-15} \mathrm{M}_{\odot} \mathrm{yr}^{-1}$ on the abundance anomalies that parameter free evolution leads to.

\subsection{A AND F STARS}

The effect of mass loss in A stars has been modeled by Michaud et al. (1983) and Michaud and Charland (1986). A mass loss rate of $310^{-16}$ to $10^{-15} \mathrm{M}_{\odot} \mathrm{yr}^{-1}$ reduces the overabundances to observed values in AmFm stars for iron peak elements as well as rare earths without perturbing the agreement for underabundant elements. The effect of mass loss was calculated on only a few elements and it is not yet possible to use the large number of abundances determined for some individual stars to constrain the model. Such a detailed comparison requires a precise determination of radiative accelerations for many elements down to a mass fraction of the star of $10^{-5} \mathrm{M}_{\odot}$ since the abundances that appear at the surface after $10^{9} \mathrm{yr}$ were originally at such depths. Calculations of the time evolution of the abundances for many elements will only become justified when such radiative accelerations are available.

It was also concluded by Michaud and Charland that a mass loss rate of $10^{-13} \mathrm{M}_{\odot} \mathrm{yr}^{-1}$ could lead to the appearance of the $\lambda$ Bootis phenomenon.

The same mass loss rate as suggested above for the AmFm stars reduces the overabundance of heavy elements $(e . g . \mathrm{Mn})$ in the $\mathrm{F}$ stars where the Li gap is measured. This is in agreement with observations (Tomkin Lambert and Balachandran 1985; Boesgaard and Lavery 1986; Thévenin, Vauclair and Vauclair 1986) that there are no apparent abundance anomalies of heavy elements in those $\mathrm{F}$ stars where $\mathrm{Be}$ is observed to be underabundant.

In these calculations, the wind is assumed to have the same composition as the convection zone. This is the simplest assumption but may not be justified. Michaud et al. (1987) evaluated that there could be separation in the wind (i. $e$. above the photosphere) for mass loss rates as high as $10^{-12} \mathrm{M}_{\odot} \mathrm{yr}^{-1}$ but this rested on approximations for the wind model that maximize the separation for a given mass loss rate. They concluded that the separation was more likely to occur in the photosphere than above. The potential effects of separation above the photosphere are neglected here.

\subsection{HELIUM STARS}

The study of He rich stars followed that of Vauclair (1975) who had suggested that mass loss might be responsible for the appearance of overabundances of He in stars with $T_{\text {eff }}>19000$ 
K. The observation of mass loss in at least some He rich stars (Barker et al. 1982) supports this suggestion. Michaud et al. (1987) emphasized that this required the mass loss rate to decrease as the $T_{\text {eff }}$ increased from 19000 to $25000 \mathrm{~K}$ : the larger ionisation of He leads to a smaller diffusion velocity. This may be understood if the separation takes place where the magnetic field is horizontal so that it can reduce the mass loss rate (see also Shore 1987). The He overabundances would occur mainly where the magnetic field lines are horizontal and not at the poles. The mass loss would occur at the pole. The mass loss rate required to produce He overabundances does not allow any $\mathrm{C}, \mathrm{N}, \mathrm{O}$ separation so that these elements are expected to be normal throughout the surface (Michaud et al. 1987). However, in a nuclear physics model for the overabundance of $\mathrm{He}$, overabundances of $\mathrm{N}$ and underabundances of $\mathrm{C}$ and $\mathrm{O}$ are expected. The combined observation of these various abundances so determines the origin of $\mathrm{He}$ anomalies. The normal abundance of $\mathrm{CNO}$ would be the signature of separation taking place in the atmosphere of a star that loses mass at a rate of order $10^{-12} \mathrm{M}_{\odot} \mathrm{yr}^{-1}$ at the poles. Hunger (1986) concludes that the CNO abundances are essentially normal in the He-rich stars where they have been determined with enough accuracy.

The recent observations of Bohlender and Landstreet (1990) suggest that $\mathrm{He}$ would be concentrated close to the poles in HD 64740. This could only be understood if the wind were relatively small at the poles in this star, smaller than needed to reproduce the line profiles of $\sigma$ Ori $\mathrm{E}$, for instance. The $\mathrm{N}$ and $\mathrm{O}$ abundaces are nearly uniform while $\mathrm{Si}$ and $\mathrm{Mg}$ seem concentrated in a band. The average abundances are approximately solar once NLTE corrections are included. The model of Hunger, Heber and Groote (1989) is incompatible with the model described above mainly in that it requires a mass loss rate of $10^{-9} \mathrm{M}_{\odot} \mathrm{yr}^{-1}$ at the two He rich caps. Otherwise it makes the interesting suggestion that He rich caps may appear to trail behind the magnetic pole because of the effect of rotation on particle trajectories and how these may follow field lines. Since He-rich stars are relatively rapid rotators, rotation may well play a role, either through the wind model or through meridional circulation.

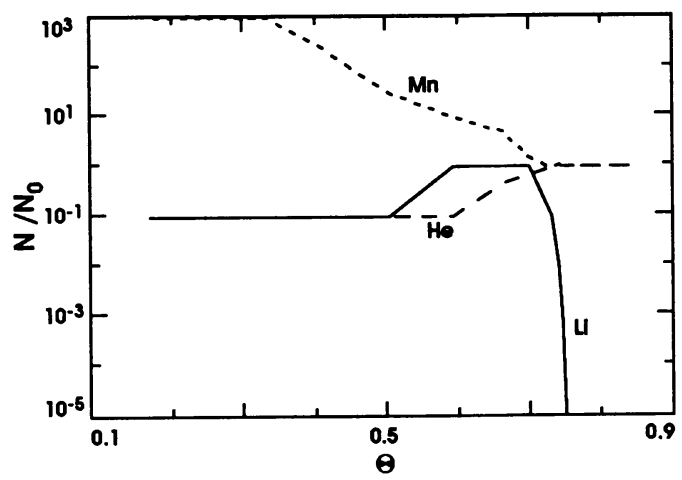

Figure 3. Same as Fig. 1 but in the presence of meridional circulation, for an equatorial rotation velocity of $50 \mathrm{~km} \mathrm{~s}^{-1}$. There is no abundance anomaly at $\theta>0.8$, except the $\mathrm{Li}$ underabundance, due to the burning of $\mathrm{Li}$. It appears at approximately the same $\mathrm{T}_{\text {eff }}$ as the $\mathrm{Li}$ gap is observed. The Mn abundance is affected by rotation mainly below $\mathrm{T}_{\text {eff }}=8000 \mathrm{~K}(\theta>$ 0.7). For $\theta<0.7$, the $\mathrm{He}$ and $\mathrm{Li}$ underabundances, while still present, are much smaller than in the absence of rotation. All effects of diffusion are wiped out for $T_{\text {eff }}<7000 \mathrm{~K}$. For $T_{\text {eff }}$ $<6600 \mathrm{~K}$, the observed equatorial rotation velocity is smaller than $50 \mathrm{~km} \mathrm{~s}^{-1}$ and the meridional circulation used here is an overestimate. 


\subsection{SOLAR TYPE STARS}

A number of authors (Weymann and Sears 1965; Hobbs, Iben and Pilachowski 1989; Boothroyd, Sackmann and Fowler 1990) have suggested that the Li depletion in solar type stars could be due to mass loss. A mass loss rate 500 times the current one would have been sufficient and it could have occurred early in the solar evolution. However, Swenson and Faulkner (1989) conclude that this requires most Hyades $G$ dwarfs to have descended from an extremely narrow range of initial masses $\left(1.08 \pm 0.01 \mathrm{M}_{\odot}\right)$. No star with smaller initial mass could have formed.

\section{Rotating stars}

Meridional circulation can now be calculated from first principles using the Tassoul and Tassoul (1982) solution. It allows the introduction of rotation into stellar models without using arbitrary parameters. The abundance anomalies predicted then become a test of the model for meridional circulation (see Fig. 3).

\subsection{A AND F STARS: COMPETITION WITH ATOMIC DIFFUSION}

Approximating meridional circulation by a two column flow, Michaud (1982) obtained that the gravitational settling of $\mathrm{He}$ should lead to the disappearance of the superficial convection zone in $\mathrm{HgMn}$ and AmFm stars only for rotational velocities of $100 \mathrm{~km} \mathrm{~s}^{-1}$ or less. This agrees with observations of an upper limit for the rotational velocity at which the HgMn and AmFm phenomena are observed (see \$3.1). This result was later confirmed by Charbonneau and Michaud (1988a) using detailed bi-dimensional solutions of the transport equations. The evolutionnary calculations were stopped after $10^{6} \mathrm{yr}$ or so because of numerical instabilities. They were extended by a factor of 10 by Charbonneau and Michaud (1991; see their Fig. 2) using a finite element solution of the transport equation. That meridional circulation leads naturally to the limiting equatorial rotation velocity of about $100 \mathrm{~km} \mathrm{~s}^{-1}$ for the $\mathrm{HgMn}$ and AmFm stars is a strong argument in favour of the description of global motions in rotating stars by meridional circulation as calculated by Tassoul and Tassoul (1982).

Charbonneau and Michaud (1991) calculated the effect of meridional circulation on the overabundances of heavy elements produced by radiation driven diffusion both for AmFm and $\lambda$ Booti stars. It is difficult to follow numerically particle transport of heavy elements in presence of meridional circulation. The rapid variation of the radiative acceleration close to the bottom of the convection zone imposes stiff constraints. The required grid is shown in Fig. 1 of Charbonneau and Michaud (1991). Typically 150 radial by 30 azimuthal elements were necessary.

For Am stars, once the He convection zone has disappeared, the diffusion of heavy elements is virtually unaffected by meridional circulation (Fig. 5 of Charbonneau and Michaud 1991). Below the $\mathbf{H}$ convection zone, diffusion is much more rapid than below the $\mathrm{He}$ convection zone so that, once meridional circulation is slow enough for the settling of He to occur, it then has no effect on the diffusion of heavy elements, at least of all those considered. The situation is more interesting below the He convection zone for those stars rotating fast enough $\left(V_{e}>100 \mathrm{~km} \mathrm{~s}^{-1}\right)$ for the He convection zone not to disappear (Fig. 6 of Charbonneau and Michaud 1991). Then, there do appear overabundances of a few elements, such as Mn and 
$\mathrm{Eu}$, by factors of approximately 5 for velocities up to $200 \mathrm{~km} \mathrm{~s}^{-1}$. Even in rapidly rotating stars, abundance anomalies are expected. They should be compared to the observed variations in the so called normal stars (see the review by Cowley in this volume).

For the $\lambda$ Bootis stars the results presented on Fig. 6 of Charbonneau and Michaud (1991) show that overabundances produced by diffusion below the He convection zone do not transform into underabundances as time passes. It does not appear that gravitational settling in the presence of meridional circulation can produce the $\lambda$ Booti phenomenon without adding mass loss (see Michaud and Charland 1986). However, these results show that diffusion can have large effects on the abundance of heavy elements for rotational velocities up to $200 \mathrm{~km} \mathrm{~s}^{-1}$. Because the diffusion velocity of heavy elements is often larger than that of $\mathrm{He}$, meridional circulation can, under certain circumstances, eliminate the effects of the settling of $\mathrm{He}$ but not of the diffusion of heavy elements.

Charbonneau and Michaud (1988b) extended these results to lower mass stars assuming that meridional circulation penetrated the convection zone (see $\$ 2$ above). The limiting velocity allowing $\mathrm{He}$ and $\mathrm{Li}$ settling decreases rapidly with $\mathrm{T}_{\text {eff }}$. With $\alpha=1.4$ and Cox and Steward opacities, the limiting equatorial velocity is $50 \mathrm{~km} \mathrm{~s}^{-1}$ at $\mathrm{T}_{\text {eff }}=7260 \mathrm{~K}$ and $13 \mathrm{~km} \mathrm{~s}^{-1}$ at $\mathrm{T}_{\text {eff }}=$ $6920 \mathrm{~K}$. This comes, as $T_{\text {eff }}$ decreases, from the increase of the mass in the convection zone and so the increase of $\rho$ at its bottom. While the meridional circulation velocity hardly varies as the density increases, the diffusion velocity varies approximately as $\rho^{-0.5}$. As $\mathrm{T}_{\text {eff }}$ decreases, the ratio of the meridional circulation velocity to the diffusion velocity increases for a given equatorial velocity, leading to a smaller critical equatorial rotation velocity. This implies that gravitational settling could not cause the Li gap in those stars of the Hyades that rotate faster than $13 \mathrm{~km} \mathrm{~s}^{-1}$. This is the case for most Hyades stars with small Li abundance in the gap (see Fig. 2.10 of Michaud and Charbonneau 1990).

These calculations do not take into account the possible effect of the settling of $\mathrm{He}$ on meridional circulation (Chahbenderian 1984). In the central solar regions, the $\mu$ gradient reduces meridional circulation by orders of magnitude (Mestel 1965; Tassoul and Tassoul 1984). The $\mu$ gradient caused by He settling might also reduce meridional circulation so that the results obtained up to now might overestimate the effects of meridional circulation once $\mathrm{He}$ has started to settle.

It has also been suggested that meridional circulation leads to a two cell circulation pattern and that this may have important effects on the $\mathrm{Li}$ abundance in main sequence stars (see Vauclair this volume for a review). The existence of such a double cell is currently challenged since only in barotropic models has it been shown to exist (Tassoul 1990) and rotating stars are not barotropes. If real, double cells should appear in the Tassoul and Tassoul solution when second order terms are included; such solutions will be of great interest.

\subsection{F STARS: ADVECTION OF NUCLEAR PROCESSED MATTER}

Meridional circulation modifies superficial abundances by bringing to the surface matter that has been processed in the interior by nuclear reactions. This could lead to the $\mathrm{Li}$ gap by bringing to the convection zone matter that has been depleted from its $\mathrm{Li}$ (see Fig. 5.2 of Michaud and Charbonneau 1990). The burning of $\mathrm{Li}$, by the $(\mathrm{p}, \alpha)$ reaction, occurs at $\mathrm{T}=2.6$ $10^{6} \mathrm{~K}$. When, after a time $t_{0}$, matter that was originally deeper than $\mathrm{T}=2.610^{6} \mathrm{~K}$ arrives in the convection zone, the $\mathrm{Li}$ abundance starts decreasing there. It then decreases exponentially with a time constant $\theta$, equal to the time it takes to replace the mass of the convection zone by new material: 


$$
c=c_{0} \exp \left(\left(t-t_{0}\right) / \theta\right) \quad \text { for } t>t_{0} \text {. }
$$

Both $\theta$ and $t_{0}$ are inversely proportional to the square of the rotation velocity. The effect of diffusion was completely neglected in this calculation. When the radiative acceleration on $\mathrm{Li}$ is smaller than gravity, the settling increases the effect of $\mathrm{Li}$ burning and so taking it into account could only increase the underabundance. When, however, radiative acceleration is larger than gravity, the surface $\mathrm{Li}$ is maintained in the convection zone by the upward diffusion and so shielded from transport into the burning zone (see Figure $5.1 \mathrm{~b}$ of Michaud and Charbonneau 1990) so long as the upward diffusion velocity is larger than the meridional circulation velocity which, for stars with $T_{\text {eff }}>6900 \mathrm{~K}$, occurs if $\mathrm{V}<50 \mathrm{~km} \mathrm{~s}^{-1}$,

Fig. 5.3 of Michaud and Charbonneau (1990) shows the Li abundances to be expected from such a model at the age of the Hyades and of UMa. The equatorial rotational velocity used is an envelope to the observed rotational velocities of stars of the Hyades where Li was measured (see Fig. 5.1 of Michaud and Charbonneau 1990). The agreement is satisfactory for the Hyades. A somewhat larger rotational velocity would be needed to explain the results for UMa. In both the model based on gravitational settling (see § 3 ) and that based on meridional circulation that we just described, the $\mathrm{T}_{\text {eff }}$ at which the $\mathrm{Li}$ gap occurs is explained by the drop in the $g_{R}(\mathrm{Li})$ below the convection zone for $\mathrm{T}_{\text {eff }}<6900 \mathrm{~K}$, as described in $\S 2$.

It should be possible to distinguish between these two models by observations of $O$ and $\mathrm{N}$. Underabundances of these elements are expected in the model based on diffusion but not on that involving burning. However more calculations of $g_{R}(O)$ and $g_{R}(N)$ are needed to confirm this test. Observations of Be abundances in cluster giants would also put useful constraints on these models (see Charbonneau, Michaud and Proffitt 1989).

Atomic diffusion explains the presence of the $\mathrm{Li}$ gap in slowly rotating stars and meridional circulation explains it in the more rapidly rotating stars of the Hyades in the gap. The two models may be complementary. More detailed calculations are needed to confirm how stars rotating at intermediate velocities are affected. A detailed understanding of how the presence of a convection zone modifies meridional circulation at its base is also needed (see $\$ 2$ above).

\subsection{EFFECT ON THE SUBGIANT AND GIANT BRANCHES}

These calculations were extended by Charbonneau, Michaud and Proffitt (1989) to predict Li depletion on the giant branch. Standard stellar evolution implies very little main sequence destruction of $\mathrm{Li}$ and predicts that, as stars evolve on the giant branch, the $\mathrm{Li}$ remaining after the pre-main sequence is diluted as the superficial convection zone becomes deeper. Meridional circulation increases the mass fraction of the star where $\mathrm{Li}$ is destroyed. The result depends only on the rotation velocity and on no arbitrary parameter. Meridional circulation can lead to extreme $\mathrm{Li}$ underabundances in first ascent giants. In the mass range $1.2<\mathrm{M}<2.0 \mathrm{M}_{\odot}$, stars having equatorial rotation velocities larger than $30-35 \mathrm{~km} \mathrm{~s}^{-1}$ destroy most of their $\mathrm{Li}$ on the main sequence. The results of Charbonneau, Michaud and Proffitt (1989) fit reasonably well the observed Li abundances in the Giants of NGC 752 and M67 but not those of NGC 7789. The results for this latter cluster are rather consistent with the standard evolution that implies very little main sequence depletion of $\mathrm{Li}$. To do their calculations, Charbonneau, Michaud and Proffitt (1989) used a maxwellian distribution of rotation velocities scaled according to the observed relation between $v$ sin $i$ and stellar mass. Some observations, however, suggest an excess of slow rotators at least in early type stars (see for instance Wolff, 
Edwards and Preston 1982). A large excess of slow rotators in NGC 7789 could lead to a larger Li abundance for most stars. This is, however, an ad hoc hypothesis.

\section{Turbulence}

Models based on turbulent transport have been discussed elsewhere in this volume and need not be reviewed here. We only discuss the upper limits to turbulence that the models described above can tolerate. This is specially appropriate since the meridional circulation model of Tassoul and Tassoul requires some turbulent viscosity to be internally consistent (see the last paragraph of Charbonneau and Michaud 1988a and Tassoul and Tassoul 1986, 1989, for a discussion of the turbulent viscosity needed and of the implications for the turbulent diffusion coefficient).

For AmFm stars, Vauclair, Vauclair and Michaud (1978) determined upper limits to turbulence in the absence of meridional circulation by requesting that He settle sufficiently for the disappearance of the He II convection zone. They obtained that the turbulent diffusion coefficient had to be smaller than 500 times the atomic diffusion coefficient below the $\mathrm{He}$ convection zone. The exact value of this factor is a function of the dependence of turbulence on density (or radius). While Charbonneau and Michaud (1991) confirmed that result, they also showed that, in the presence of meridional circulation, turbulence had to be further reduced by a factor of about 50 for the He convection zone to disappear in Am stars with an equatorial rotation velocity of $75 \mathrm{~km} \mathrm{~s}^{-1}$ (see their Fig. 9a). The acceptable upper limit becomes merely a factor of 10 larger than the atomic diffusion coefficient. The upper limit would be larger in more slowly rotating stars.

Proffitt and Michaud (1991; see also $\$ 3.3$ above) similarly conclude that the maximum turbulent diffusion coefficient that is allowed in Halo star models without destroying too much of the original $\mathrm{Li}$ is about a factor of $\mathbf{1 0}$ larger than the atomic diffusion coefficient.

\section{Horizontal branch}

Field subdwarfs are known to have He underabundances (Sargent and Searle 1968) and, where the horizontal branch crosses the main sequence, to have anomalies similar to those of peculiar stars of the same $T_{\text {eff }}(e . g$. observations of Feige 86 by Sargent and Searle 1967 and Baschek and Sargent 1976). There is observational evidence that subdwarf stars are the continuation of the horizontal branch and that all of them have He underabundances, if they are hot enough for He to be observed (Hunger et al. 1981; Baschek et al. 1982; Heber 1986). Detailed NLTE $\mathrm{g}_{\mathrm{R}}(\mathrm{He})$ have been calculated for these objects (Michaud et al. 1989) and have confirmed that the observed underabundances are explained by gravitational settling. However radiative accelerations remain smaller than gravity at the observed He underabundances, so that they cannot prevent further He settling. Some other process must be preventing further settling. As on the main sequence (see §4), mass loss could well be involved. Mass loss has already been suggested to explain the large $\mathrm{Si}$ underabundances in some horizontal branch stars (Michaud et al. 1985). Using more detailed radiative accelerations for those objects Bergeron et al. (1988) confirmed that result.

Michaud, Vauclair and Vauclair (1983) calculated particle transport along the horizontal branch and concluded that anomalies could appear for $T_{\text {eff }}>8000 \mathrm{~K}$, but are likely to appear 
only above $11000 \mathrm{~K}$ because competing processes probably dominate in the cooler stars. Comparing these results with observations of field horizontal branch stars is inconclusive since these have generalized underabundances that vary from star to star, so that it is impossible to know the original abundances of the observed objects. Glaspey et al. (1989) carried out observations of horizontal branch stars in the metal poor cluster NGC 6752. They found an overabundance by a factor of 50 for $\mathrm{Fe}$ (compared to the cluster original abundance) in the hotter star they observed $\left(T_{\text {eff }}=16000 \mathrm{~K}\right)$ but not in the cooler one $\left(T_{\text {eff }}=10000 \mathrm{~K}\right)$. These observations involved only a few elements, in only two stars of one cluster. Given the presumably uniform original abundances of heavy elements in clusters, it would be possible to obtain the $T_{\text {eff }}$ dependence of the anomalies and so important constraints on the hydrodynamics and the evolutionary status of these objects. Observations of additional stars and clusters are needed.

\section{REFERENCES}

Alecian, G., and Artru, M.-C., 1990, Astron. Astrophys., in press.

Alecian, G., and Michaud, G. 1981, Astrophys. J., 245, 226.

Barker, P., K., Brown, D. N., Bolton, C. T., and Landstreet, J. D. 1982, in Advances in Ultraviolet Astronomy: Four Years of IUE Research, NASA CP-2238, p. 589.

Baschek, B., Kudritzki, R. P., Scholz, M., and Simon, K. P. 1982, Astron. Astrophys., 108, 387.

Baschek, B., and Sargent, A. I. 1976, Astron. Astrophys., 53, 47.

Bergeron, P., Wesemael, F., Michaud, G., and Fontaine, G. 1988, Astrophys. J., 332, 964.

Boesgaard, A. M., and Lavery, R. J. 1986, Astrophys. J., 309, 762.

Bohlender, D. A., and Landstreet, J. D. 1990, Astrophys. J., 358, 274.

Boothroyd, A. I., Sackmann, I.-J., and Fowler, W. A. 1990, Astrophys. J., submitted.

Chahbenderian, M. 1984, Thèse de troisième cycle, Université de Paris VII.

Chapman, S., and Cowling, T. G. 1970, The Mathematical Theory of non-uniform Gases (3d ed.; Cambridge: Cambridge University Press).

Charbonneau, P., and Michaud, G. 1988a, Astrophys. J., 327, 809.

Charbonneau, P., and Michaud, G. 1988b, Astrophys. J., 334, 746.

Charbonneau, P., and Michaud, G. 1991, Astrophys. J., submitted for publication.

Charbonneau, P., Michaud, G., and Proffitt, C. R. 1989, Astrophys. J., 347, 821.

Cox, A. N., and Stewart, J. N. 1970, Astrophys. J. Suppl., 19, 243.

Deliyannis, C. P., Demarque, P., and Kawaler, S. D. 1990, Astrophys. J. Suppl., in press.

Glaspey, J. W., Michaud, G., Moffat, A. F. J., and Demers, S. 1989, Astrophys. J., 339, 926.

Heber, U. 1986, Astron. Astrophys., 155, 33.

Hobbs, L. M., Iben, I. Jr, and Pilachowski, C. 1989, Astrophys. J., 347, 817.

Hunger, K. 1986, in Hydrogen Deficient Stars and Related Objects, IAU Colloquium 87, Ed. K. Hunger, D. Schonberner and N. K. Rao (Dordrecht: Reidel), p. 261.

Hunger, K., Gruschinske, J., Kudritzki, R. P., and Simon, K. P. 1981, Astron. Astrophys., 95, 244.

Hunger, K., Heber, U., and Groote, D. 1990, in Intrinsic Properties of Hot Luminous Stars, $P$. A. S. P. Conference Series, in press.

Loeb, A., Bahcall, J. N., and Milgrom, M. 1989, Astrophys. J., 341, 1108.

Lumer, E., Forestini, M., and Arnould, M. 1989, preprint. 
Mestel, L. 1965, in Stars and Stellar Systems, Vol. 8, Stellar Structure, ed. L. H. Aller and D. B. McLaughlin (Chicago: University of Chicago Press), p. 465.

Michaud, G. 1977, Nature, 266, 433.

Michaud, G. 1982, Astrophys. J., 258, 349.

Michaud, G, 1986, Astrophys. J., 302, 650.

Michaud, G. 1987, Physica Scripta, 36, 112.

Michaud, G. 1991, Annales de Physique, submitted for publication.

Michaud, G., and Babel, J. 1991, in Stellar Atmospheres: Beyond Classical Models, NATO Advanced Research Workshop, Ed. L. Crivellari et al. (Doldrecht: Kluwer), in press.

Michaud, G., Bergeron, P., Heber, U., and Wesemael, F. 1989, Astrophys. J., 338, 417.

Michaud, G., Bergeron, P., Wesemael, F., and Fontaine, G. 1985, Astrophys. J., 299, 741.

Michaud, G., and Charbonneau, P. 1990, Space Science Reviews, in press.

Michaud, G., and Charland, Y. 1986, Astrophys. J., 311, 326.

Michaud, G., Charland, Y., Vauclair, S., and Vauclair, G. 1976, Astrophys. J., 210, 447.

Michaud, G., Dupuis, J., Fontaine, G., and Montmerle, T. 1987, Astrophys. J., 322, 302.

Michaud, G., Montmerle, T., Cox, A.N., Magee N.H., Hodson, S.W., and Martel, A. 1979, Astrophys. J., 234, 206.

Michaud, G., Tarasick, D., Charland, Y., and Pelletier, C. 1983, Astrophys. J., 269, 239.

Michaud, G., Vauclair, G., and Vauclair, S. 1983, Astrophys. J., 267, 256.

Paquette, C., Pelletier, C., Fontaine, G., and Michaud, G. 1986, Astrophys. J. Suppl., 61, 177.

Pelletier, C., Fontaine, G., Wesemael, F., Michaud, G., and Wegner, G. 1986, Astrophys. J., 307, 242.

Proffitt, C. R., and Michaud, G. 1991, Astrophys. J.

Richer, J., Michaud, G., and Proffitt, C. 1991, Astrophys. J. Suppl., submitted for publication.

Sargent, W. L. W., and Searle, L. 1967, Astrophys. J. Letters, 150, L33.

Sargent, W. L. W., and Searle, L. 1968, Astrophys. J., 152, 443.

Schatzman, E. 1969, Astron. Astrophys., 3, 331.

Shore, S. N., 1987, Astron. J., 94, 731.

Swenson, F. J., and Faulkner, J. 1989, B. A. A. S., 21, 1101.

Tassoul, J.-L. 1990, in Angular Momentum and Mass Loss for Hot Stars, eds. L. A. Wilson and R. Stalio (Dordrecht: Kluwer), 7.

Tassoul, J.-L., and Tassoul, M. 1982, Astrophys. J. Suppl., 49, 317.

Tassoul, J.-L., and Tassoul, M. 1986, Geophys. Astr. Fluid Dyn., 36, 303.

Tassoul, J.-L., and Tassoul, M. 1989, Astron. Astrophys., 213, 397.

Tassoul, M., and Tassoul, J.-L. 1984, Astrophys. J., 279, 384.

Thévenin, F., Vauclair, S., and Vauclair, G. 1986, Astron. Astrophys., 166, 216.

Tomkin, J., Lambert, D. L., and Balachandran, S. 1985, Astrophys. J., 290, 289.

Vauclair, G., Vauclair, S., and Pamjatnikh, A. 1974, Astron. Astrophys., 31, 63.

Vauclair, S. 1975, Astron. Astrophys., 45, 233.

Vauclair, S. 1987, in Atmospheric Diagnostics of Stellar Evolution: Chemical Peculiarity, Mass Loss, and Explosion, IAU Colloquium No. 108., Ed. K. Nomoto (Berlin: Springer Verlag) p. 13.

Weymann, R., and Sears, R. L. 1965, Astrophys. J., 142, 174.

Wolff, S. C., Edwards, S., and Preston, G. W. 1982, Astrophys. J., 252, 322. 